\title{
Gastrointestinal Stenting
}

National Cancer Institute

\section{Source}

National Cancer Institute. Gastrointestinal Stenting. NCI Thesaurus. Code C116572.

The placement of a mesh tube anywhere within the gastrointestinal tract. It is used to keep the lumen open and free of obstruction. 